\title{
Comparative Evaluation of Drill-Free and Self-Tapping Titanium Miniscrews for Semi-Rigid Internal Fixation in Maxillofacial Trauma
}

\author{
Namitha M. Hiriyanna $^{1}$ (D) Saikrishna Degala ${ }^{1} \cdot$ Sujeeth Kumar Shetty $^{1}$
}

Received: 10 March 2021 / Accepted: 5 August 2021/Published online: 18 August 2021

(C) The Association of Oral and Maxillofacial Surgeons of India 2021

\begin{abstract}
Introduction Conventional semi-rigid fixation systems in maxillofacial trauma use self-tapping titanium miniscrews (STS) that require preliminary drilling of a pilot hole. Although drill-free miniscrews (DFS) accompany these systems, they have not dominated practice despite their allure of improved screw-bone contact and holding power. The COVID-19 pandemic has brought these DFS to light as they avoid aerosol production. The present study has compared DFS to STS in patients being treated with miniplate fixation for maxillofacial trauma to understand their feasibility for maxillofacial fracture fixation.

Methodology This prospective case-control study sampled 16 patients each with zygomaticomaxillary buttress fracture and parasymphysis fracture of the mandible and grouped alternating patients as case (DFS) and control (STS). Intraoperatively duration of fixation, incidence of screw failures and fragment stability; postoperatively occlusion, neurosensory deficits, teeth vitality and infection and removal rates were evaluated at postoperative week 1 , $3,6,12$ and 24 using Cramer's V test. A $P$ value $<0.05$ was considered significant.

Results In the 32 patients evaluated, DFS reduced internal fixation time at zygomaticomaxillary buttress $(P=0.001)$
\end{abstract}

Namitha M. Hiriyanna

namithamh@hotmail.com

Saikrishna Degala

degalasaikrishna@gmail.com

Sujeeth Kumar Shetty

shettymaxfax@gmail.com

1 Oral and Maxillofacial Surgery, JSS Dental College, JSS

Academy of Higher Education and Research,

Mysuru 570015, India but not at parasymphysis $(P=0.206)$. No significant difference in screw failures or fragment stability was observed. Stable occlusion was maintained in all groups with vital teeth and intact neurosensory function, but the summative incidence of infection was significant at week 24 when STS was used at parasymphysis $(P=0.019)$.

Discussion While DFS may facilitate ease of insertion with a single instrument pick-and-screw-in approach, avoiding thermal osteonecrosis and aerosol production, they fail to confer any other clinical advantage.

Keywords Miniscrews · Osteosynthesis · Self-drilling · Self-tapping $\cdot$ Maxillofacial trauma $\cdot$ Drill-free screw

\section{Introduction}

Open reduction and internal fixation of maxillofacial fractures have become an everyday procedure in modernday surgical practice. Miniplate osteosyntheses consist of miniplates that are secured to bone fragments with miniscrews that are conventionally self-tapped after preliminary preparation of a pilot hole using drill bits of diameter equal to that of the screw's core. Rotational torque given at the screw head advances it axially, while the flutes engaging into the surrounding bone. Although self-tapping screws (STS) are universal in maxillofacial plating modules, predrilling has been understood to cause inadvertent thermal necrosis of adjacent bone [1, 2], strip thin cortical areas of the midface [3] and add an unavoidable burden of increased instrumentation.

Drill-free screws (DFS) have a tapered core with threads that begin at the tip, which are guided along an axis of rotation till the screw head, enabling them to be self-drilling and self-tapping at the same time (Fig. 1). 


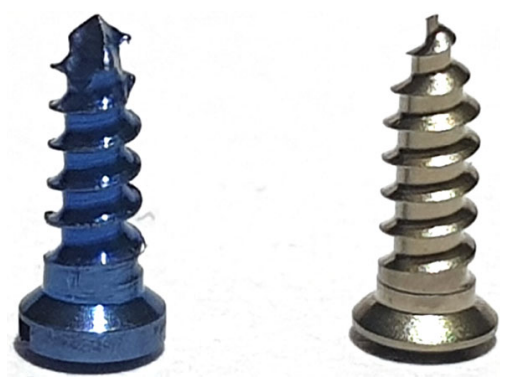

Fig. $12 \mathrm{~mm}$ diameter and $6 \mathrm{~mm}$ length self-tapping miniscrew (left) and drill-free miniscrew (right)

Ultrastructural studies demonstrate better screw-bone contact in DFS in comparison with STS, with better holding power, surrounded by biologically active bone debris $[3,4]$. The actual gain from DFS may only seem theoretical as the facial skeleton is an assembly of bones with non-uniform cortical thicknesses and increased insertional torque may cause more harm in a practical scenario [5]. The present study has compared the intraoperative and postoperative performance of DFS to STS, in patients being treated with miniplate fixation for fractures of zygomaticomaxillary buttress (ZMB) and parasymphysis fracture of the mandible (PSM), to understand their feasibility for maxillofacial fracture fixation.

\section{Material and Methods}

\section{Study Design}

This was a preliminary prospective case-control study that assessed the intraoperative and postoperative performance of drill-free and self-tapping titanium miniscrews in patients being treated with a miniplate fixation for fractures of the zygomaticomaxillary buttress and parasymphysis fracture of the mandible who satisfied the eligibility criteria. The study was conducted as per the Declaration of Helsinki guidelines.

\section{Study Setting.}

This study was approved by the institutional ethical committee and was conducted in a tertiary trauma centre in Mysuru city for 20 months between February 2018 and October 2019.

\section{Inclusion and Exclusion Criteria}

To be included, patients between the age of 18-50 years of either gender, who were classified ASA grade I and grade II for general anaesthesia, in whom miniplate fixation was indicated for open reduction and internal fixation of ZMB and PSM fractures, who consented for the procedure and regular recall visits were considered. Exclusion criteria included patients with systemic bone diseases such as osteoporosis, systemic disorders with hepatic, respiratory, cardiac, endocrine or metabolic impairment and patients under long-term corticosteroid or bisphosphonate therapy.

\section{Fracture Type}

The fracture type was standardised for the purpose of the study as follows:

Fracture of zygomaticomaxillary buttress-disruptions occurring in zygomaticomaxillary articulations, extending circumferentially around the anterior and posterolateral walls of the maxillary sinus.

Fracture of parasymphysis of mandible-fractures occurring within the area of the symphysis bounded by vertical lines distal to the canine teeth.

\section{Test Groups}

By purposive sampling, 16 patients with ZMB fracture and 16 patients with PSM fracture were considered, and among those 16 patients, every consecutive sampled patient was alternatively grouped into either case (DFS) or control (STS) group.

Group ZMB-DFS was the case study group consisting of 8 patients who were treated using an L-shaped titanium miniplate with 4 titanium DFS. Group ZMB-STS was the control group of 8 patients who were similarly treated with 4 titanium STS. Group PSM-DFS was the case study group consisting of 8 patients who were treated using two 4-holed titanium miniplates with 8 titanium DFS. Group PSM-STS was the control group of 8 patients who were similarly treated with 8 titanium STS.

\section{Pre-Surgical Assessment}

Patients were diagnosed by clinical examination with radiological evidence of fracture on computed tomography scan. All patients underwent routine blood workup for a general anaesthetic procedure.

\section{Surgical Procedure}

All patients were operated on by the same surgeon and operation theatre team. Following nasoendotracheal intubation and aseptic precautions, ZMB fractures were approached through a maxillary buccal vestibular incision and PM fractures were approached through an intraoral lower labial vestibular incision to expose the fracture site. Post fracture reduction and plate adaptation, STS were 
placed into pilot holes drilled using $1.8 \mathrm{~mm}$ drill bits, with copious saline irrigation, with drill bits ensured to have not been used more than three times. DFS were screwed directly into the bone with manual insertional torque through a friction grip screwdriver without saline irrigation. All screws used were of the same diameter of $2 \mathrm{~mm}$ and length of $6 \mathrm{~mm}$. The surgical site was copiously irrigated with $5 \%$ povidone-iodine solution and closed using 3.0 absorbable polyglactin 910 continuous locking suture.

\section{Postoperative Management}

Patients were postoperatively administered intravenous Amoxicillin Clavulanic acid combination of $1.2 \mathrm{~g}$ and Metronidazole $500 \mathrm{mg}$ for five doses, 8th hourly, intramuscular diclofenac $75 \mathrm{mg}$ as an analgesic, and continued on oral Amoxicillin Clavulanic acid combination of $625 \mathrm{mg}$ and Metronidazole $400 \mathrm{mg}$ 8th hourly for five days on discharge. Patients were advised to use Chlorhexidine gluconate $0.2 \%$ mouthwash four times a day.

\section{Evaluation of Parameters}

\section{Intraoperative}

The duration of hardware fixation was calculated after fracture reduction from plate adaptation until the final tightening of screws as the number of minutes taken. Screw failures were defined as a fracture of the screw head or loss of primary stability of screw after tightening necessitating use of emergency screws of greater diameter. Fragment stability of fracture fragments was evaluated post-fixation after the last screw was placed, as present or absent.

\section{Postoperative}

Patients were followed up at intervals of week 1, 3, 6, 12 and 24 and assessed for occlusal stability, infection at the fracture site, neurosensory deficits, teeth vitality of three teeth on either side of the fracture line and need for removal of miniplates and miniscrews. Fracture site infection was confirmed with a positive culture of a swab sweep over the sinus opening at the operated site. Neurosensory deficits were evaluated using brush directional discrimination and two-point discrimination tests. Teeth vitality was evaluated using heat and cold sensitivity tests.

\section{Statistical analysis}

Statistical analyses were produced using SPSS version 22 for Windows software.
The intraoperative performance was compared between Group ZMB-DFS \& ZMB-STS, Group PSM-DFS \& PSMSTS and Group ZMB-DFS \& PSM-DFS and with independent samples t-test and Chi-square test. Postoperative parameters were compared between all groups using Cramer's V analyses. The significance level was set at $P<0.05$.

\section{Results}

On the whole, there were 32 patients (27 male and 5 female) allocated to 4 groups with the mean age (in years) of $31.75 \pm 13.35$ in ZMB-DFS, $33.50 \pm 10.06$ in ZMBSTS, $26.88 \pm 5.96$ in PSM-DFS and $36.13 \pm 9.73$ in PSM-STS. The age was matched between groups, but gender was not matched.

The average internal fixation time (in minutes) at ZMB with DFS was $4.5 \pm 1.4$ and with STS was $7.62 \pm 1.7$, and at PSM with DFS was $10.35 \pm 1.05$ and with STS was $11 \pm 2.2$. DFS produced a significant $(P=0.001)$ reduction in internal fixation time at ZMB, while the time-saving effect was not significant in PSM $(P=0.206)$. The incidence of screw failure was not significant when DFS and STS were compared (Table 1). When DFS was compared between ZMB and PSM regions, time reduction was significant $(P=0.001)$, while screw failures were not $(P=0.718)$. Fracture stability was $100 \%$ with no evidence of fragment mobility post-fixation in any of the patients.

All patients maintained stable occlusion postoperatively at all follow-up intervals. The influence of screw design on the incidence of neurosensory deficits (Table 2) and loss of teeth vitality (Table 3) was not found to be significant. Summative incidence of infection was significant at week $24(P=0.019)$ in Group PSM-DFS when compared to the rest of the groups (Fig. 2). There was a need for removal of plates and screws through a second surgery in these patients.

\section{Discussion}

Surgical bone screws clamp miniplates to the bone to fix bone fragments and stabilise them. Self-tapping miniscrews have a cylindrical core shaft with continuous threads of equal pitch along its length and a blunt tapered tip with a positive rake angle. A cutting groove is milled at the tip to facilitate the removal of bone debris. Drill-free miniscrews have a tapered core shaft with threads that are guided along an axis of rotation from the tip up-to-the screw head. The triangular tip provides good insertion behaviour and simultaneous space for removal of debris (Fig. 3). Animal studies have favoured drill-free screws/self-drilling screws 
Table 1 Distribution of the samples by groups and screw failure and results of Chi-square test

\begin{tabular}{|c|c|c|c|c|c|}
\hline \multirow[t]{2}{*}{ Results } & & \multicolumn{4}{|l|}{ Groups } \\
\hline & & Group ZMB-DFS & Group ZMB-STS & Group PSM-DFS & Group PSM-STS \\
\hline \multirow[t]{2}{*}{ Success } & Frequency & 31 & 30 & 61 & 62 \\
\hline & Per cent & $96.9 \%$ & $93.8 \%$ & $95.3 \%$ & $96.9 \%$ \\
\hline \multirow[t]{2}{*}{ Failure } & Frequency & 1 & 2 & 3 & 2 \\
\hline & Per cent & $3.1 \%$ & $6.2 \%$ & $4.7 \%$ & $3.1 \%$ \\
\hline Chi-square test analysis & $P=0.554$ & $P=0.648$ & & & \\
\hline
\end{tabular}

Table 2 Distribution of samples by groups and follow-up intervals for incidence of neurosensory deficits and results of Cramer's V test

\begin{tabular}{|c|c|c|c|c|c|c|c|}
\hline \multicolumn{3}{|c|}{ Follow-up intervals } & \multicolumn{4}{|l|}{ Groups } & \multirow[t]{2}{*}{$P$ value } \\
\hline & & & Group ZMB-DFS & Group ZMB-STS & Group PSM-DFS & Group PSM-STS & \\
\hline \multirow[t]{4}{*}{ Week 1} & Yes & $\mathrm{F}$ & 0 & 0 & 0 & 2 & $\mathrm{CV}=.447$ \\
\hline & & $\%$ & $0.0 \%$ & $0.0 \%$ & $0.0 \%$ & $25.0 \%$ & $p=.094$ \\
\hline & No & $\mathrm{F}$ & 8 & 8 & 8 & 6 & \\
\hline & & $\%$ & $100.0 \%$ & $100.0 \%$ & $100.0 \%$ & $75.0 \%$ & \\
\hline \multirow[t]{4}{*}{ Week 3} & Yes & $\mathrm{F}$ & 0 & 0 & 0 & 2 & $\mathrm{CV}=.447$ \\
\hline & & $\%$ & $0.0 \%$ & $0.0 \%$ & $0.0 \%$ & $25.0 \%$ & $p=.094$ \\
\hline & No & $\mathrm{F}$ & 8 & 8 & 8 & 6 & \\
\hline & & $\%$ & $100.0 \%$ & $100.0 \%$ & $100.0 \%$ & $75.0 \%$ & \\
\hline \multirow[t]{4}{*}{ Week 6} & Yes & $\mathrm{F}$ & 0 & 0 & 0 & 2 & $\mathrm{CV}=.447$ \\
\hline & & $\%$ & $0.0 \%$ & $0.0 \%$ & $0.0 \%$ & $25.0 \%$ & $p=.094$ \\
\hline & No & $\mathrm{F}$ & 8 & 8 & 8 & 6 & \\
\hline & Yes & $\%$ & $100.0 \%$ & $100.0 \%$ & $100.0 \%$ & $75.0 \%$ & \\
\hline \multirow[t]{4}{*}{ Week 12} & & $\mathrm{~F}$ & 0 & 0 & 0 & 2 & $\mathrm{CV}=.447$ \\
\hline & & $\%$ & $0.0 \%$ & $0.0 \%$ & $0.0 \%$ & $25.0 \%$ & $p=.094$ \\
\hline & No & $\mathrm{F}$ & 8 & 8 & 8 & 6 & \\
\hline & & $\%$ & $100.0 \%$ & $100.0 \%$ & $100.0 \%$ & $75.0 \%$ & \\
\hline \multirow[t]{4}{*}{ Week 24} & Yes & $\mathrm{F}$ & 0 & 0 & 0 & 0 & - \\
\hline & & $\%$ & $0.0 \%$ & $0.0 \%$ & $0.0 \%$ & $0.0 \%$ & \\
\hline & No & $\mathrm{F}$ & 8 & 8 & 8 & 8 & \\
\hline & & $\%$ & $100.0 \%$ & $100.0 \%$ & $100.0 \%$ & $100.0 \%$ & \\
\hline
\end{tabular}

(SDS) over self-tapping screws. Bone debris formed with SDS has been considered biologically active bone tissue capable of reacting with the screw and improving performance [4] with higher bone contact in both acute and chronic phase of healing $[6,7]$. A recent biomechanical and histomorphometric study has concluded that drill-free micro-screws could provide stronger stability in the maxilla than drilling micro-screws in the early phase of healing [8].

In our in-vivo case-control study, intraoperatively DFS significantly reduced the duration for internal fixation at the ZMB in comparison with STS. We find our results in contrast with Bolm et al. who through a split-mouth study found that SDS did not demonstrate a pronounced timesaving effect while fixing a 4-holed L-shaped osteosynthesis plate in Lefort I osteotomies in orthognathic surgery when compared with STS [6]. Screw failures cause a deficient clamping force of miniplates to the bone. In our study, events of screw failure such as a fracture of screw heads, loss of primary stability of screw necessitating the use of emergency screws with a wider diameter and microcrack propagation in adjacent bone were rare and isolated events and were not of significance in the usage of both STS and DFS. This was in contrast to Heidemann et al. [9] whose clinical experience with drill-free screws showed that DFS avoided screw failure that occurs due to 
Table 3 Distribution of samples by groups and follow-up intervals for the presence of vitality of teeth and results of Cramer's V test

\begin{tabular}{|c|c|c|c|c|c|c|c|}
\hline \multicolumn{3}{|c|}{ Follow-up intervals } & \multicolumn{4}{|l|}{ Groups } & \multirow[t]{2}{*}{ Cramer's V } \\
\hline & & & Group ZMB-DFS & Group ZMB-STS & Group PSM-DFS & Group PSM-STS & \\
\hline \multirow[t]{4}{*}{ Week 1} & Yes & $\mathrm{F}$ & 8 & 8 & 8 & 6 & .447 \\
\hline & & $\%$ & $100.0 \%$ & $100.0 \%$ & $100.0 \%$ & $75.0 \%$ & $p=0.94$ \\
\hline & No & $\mathrm{F}$ & 0 & 0 & 0 & 2 & \\
\hline & & $\%$ & $0.0 \%$ & $0.0 \%$ & $0.0 \%$ & $25.0 \%$ & \\
\hline \multirow[t]{4}{*}{ Week 3} & Yes & $\mathrm{F}$ & 8 & 8 & 8 & 6 & .447 \\
\hline & & $\%$ & $100.0 \%$ & $100.0 \%$ & $100.0 \%$ & $75.0 \%$ & $p=.094$ \\
\hline & No & $\mathrm{F}$ & 0 & 0 & 0 & 2 & \\
\hline & & $\%$ & $0.0 \%$ & $0.0 \%$ & $0.0 \%$ & $25.0 \%$ & \\
\hline \multirow[t]{4}{*}{ Week 6} & Yes & $\mathrm{F}$ & 8 & 8 & 8 & 6 & .447 \\
\hline & & $\%$ & $100.0 \%$ & $100.0 \%$ & $100.0 \%$ & $75.0 \%$ & $p=.094$ \\
\hline & No & $\mathrm{F}$ & 0 & 0 & 0 & 2 & \\
\hline & & $\%$ & $0.0 \%$ & $0.0 \%$ & $0.0 \%$ & $25.0 \%$ & \\
\hline \multirow[t]{4}{*}{ Week 12} & Yes & $\mathrm{F}$ & 8 & 8 & 8 & 7 & .331 \\
\hline & & $\%$ & $100.0 \%$ & $100.0 \%$ & $100.0 \%$ & $87.5 \%$ & $p=.377$ \\
\hline & No & $\mathrm{F}$ & 0 & 0 & 0 & 1 & \\
\hline & & $\%$ & $0.0 \%$ & $0.0 \%$ & $0.0 \%$ & $12.5 \%$ & \\
\hline \multirow[t]{4}{*}{ Week 24} & Yes & $\mathrm{F}$ & 8 & 8 & 8 & 8 & - \\
\hline & & $\%$ & $100.0 \%$ & $100.0 \%$ & $100.0 \%$ & $100.0 \%$ & \\
\hline & No & $\mathrm{F}$ & 0 & 0 & 0 & 0 & \\
\hline & & $\%$ & $0.0 \%$ & $0.0 \%$ & $0.0 \%$ & $0.0 \%$ & \\
\hline
\end{tabular}

Fig. 2 Graph of distribution of samples by follow-up intervals and summative infection percentage among groups

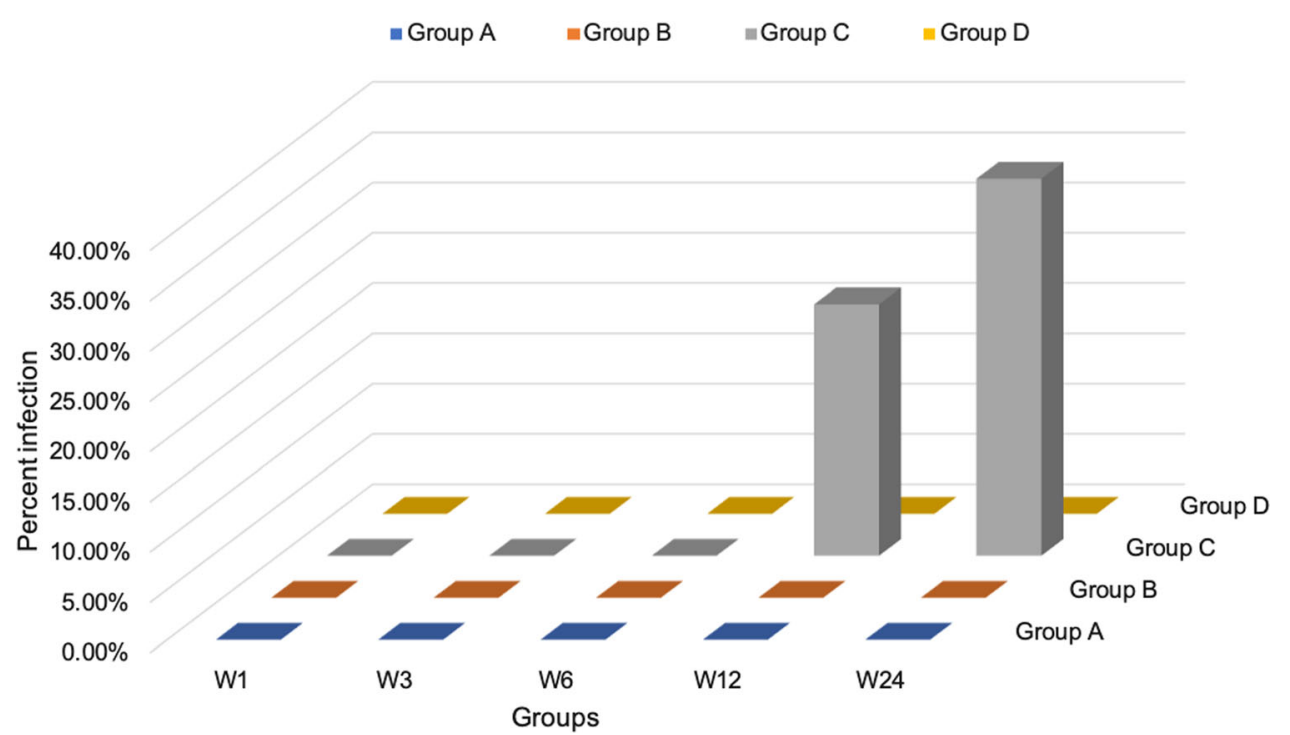

stripping of cortical bone threads in the midface when compared with STS. Our results agree with Bolm et al. [6] in that no significant decrease in the incidence of screw loosening adverse effects by DFS was found. One isolated case of minor crack propagation with the insertion of DFS that led to the separation of a triangular fragment at the inferior border of the parasymphysis region was present in our study. Prior studies have described this possibility with the use of DFS [5, 10]. This complication can arise due to increased insertional torque for DFS, which is required to overcome the friction along the thread form that may lead to microfractures in adjacent cortical bone or even exacerbate existing microfractures. The complication was dealt 

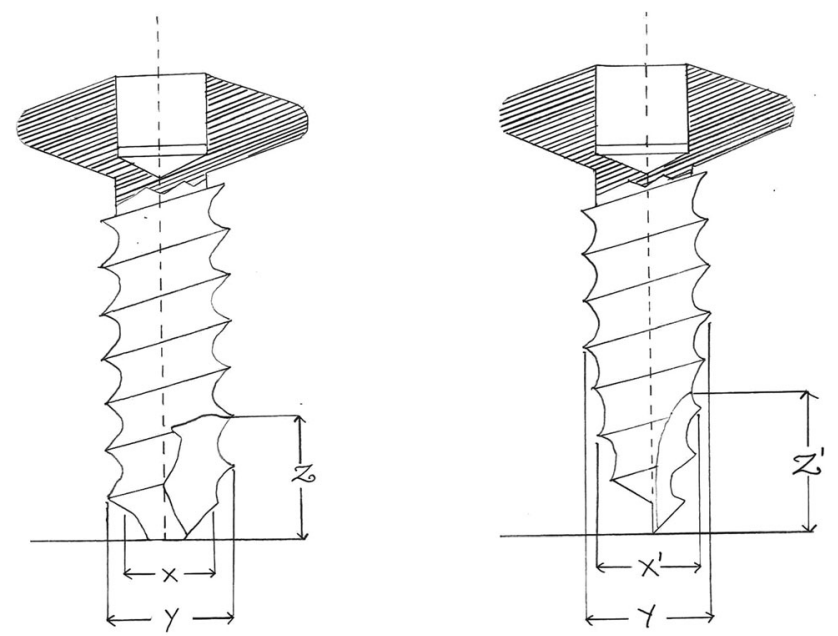

Key of image

$X$ - Core shaft with blunt tip

$X '$ - Core shaft with tapered tip

$Y$ - Diameter of screw with thread

$Z$ - Milled cutting flute of Self-tapping screws

$Z^{\prime}$ - Tapered core forming a cutting recess in Self-drilling screws

Fig. 3 Illustration of special features self-tapping miniscrew (left) and drill-free miniscrew (right)

with the removal of screws and reapplication of the plate with STS.

Initial studies have attempted to describe regions of the facial skeleton where DFS performed most efficiently and have recommended their use in the central midface, suggested possible use in the anterior mandible and lateral midface but warned against their use in mandibular angle region [9]. Clinical studies have extrapolated that conically shaped SDS performed best in cancellous bone and that STS showed best results in thick cortical bone [11, 12]. In our study, we compared the performance of DFS in two different regions of the face, the ZMB and PSM region of the mandible. The incidence of screw failures was of a similar pattern at both sites. The time duration for application of fixation was lesser at the midface than the mandible. In our clinical experience with DFS, higher insertional torque perpendicular to the cortex of the bone was required at the thicker cortical region of PSM which produced distraction of reduced fracture fragments. Additional counter stabilisation of the reduced fragments was required at the time of fixation.

In our study, postoperatively at follow-up week 24 , evidence of infection was significant $(P=0.019)$ in Group PSM-STS. There was a need for the removal of plates and screws in these three patients $(37.5 \%)$. No evidence of infection was seen in other groups. One possible explanation for this can be that when compression between screw threads and bone is inadequate, fibrous tissue stroma envelops the screw threads, leading to the screw losing its holding power and becoming loose [1] thus making it more susceptible to infection with frequent micromovements. Thermal osteolytic changes can also decrease the screwbone contact leading to screw loosening [13]. A drill bit that is used in an operating theatre for several months produces temperatures of $25.4{ }^{\circ} \mathrm{C}\left(12.4-41.3{ }^{\circ} \mathrm{C}\right)$, leading to thermal-induced bony necrosis [14]. Two cases of PSM fractures in our study, fixed with miniplates and STS showed evidence of a neurosensory deficit in the lower lip and chin on the side of the fracture till week 12 that resolved by week 24 . Widar et al. [15], Roccia et al. [16] and Dhawan et al. [17] have observed loss of teeth vitality while using self-tapping IMF screw when compared with self-drilling IMF screw. We observed no correlation of screw design of STS and DFS towards loss of vitality of teeth.

Schimming et al. while describing SDS comment on their excellent primary and long-term retention and indicate them for use in a variety of scenarios ranging from the fixation of mandibular and midface fractures, anchoring of distractors in the ascending ramus, fixation of canthal ligament or calvarial split grafts in reconstructive procedures [17]. Bolm et al. recommended SDS in areas only where drilling is difficult or impossible [6]. We agree with both the authors and perceive that the utility of self-drilling screws can be far-reaching in craniomaxillofacial surgery, especially at times where endoscopic and minimally invasive surgery is gaining popularity. DFS is also one of the ways to reduce aerosol production during surgery thus reducing surgeon and operation theatre personnel risks.

This study is limited by its lack of randomization and small sample size. More randomized-blinded clinical studies are required to evaluate surgeon experience with drill-free screws and their feasibility in all regions of the facial skeleton. Further studies are also required to investigate the use of motor-driven handpieces with adjustable torque settings, as a possible alternative to manual insertion of DFS in regions of thick cortical bone.

\section{Conclusion}

DFS may facilitate ease of insertion with a single instrument pick-and-screw-in approach, especially in the midface allowing to operate with minimal exposure while also avoiding thermal osteonecrosis and aerosol production. However, they fail to confer any other clinical advantage.

Author Contributions Conceptualization: Dr. NMH. Methodology: Dr. NMH. Formal analysis and investigation: Dr. NMH, Dr SD, Dr SKS. Writing — original draft preparation: Dr. NMH. Writing-review and editing: Dr. NMH, Dr SD, Dr SKS. Supervision: Dr SD, Dr SKS. 
Funding None

Data Availability Not Applicable

Code Availability Not applicable.

\section{Declarations}

Conflicts of interest Authors declared that they have no conflict of interest.

Ethics Approval Approved by Institutional ethical board.

Consent to Participate Informed Consent taken from all participants.

Consent for Publication Authors have given consent for publication.

\section{References}

1. Schatzker J, Horne JG, Sumner SG (1975) The effect of movement on the holding power of screws in bone. Clin Orthop Relat Res 111:257-262

2. Heidemann W, Gerlach KL, Gröbel KH, Köllner HG (1998) Drill free screws: a new form of osteosynthesis screw. J Craniomaxillofac Surg 26(3):163-168. https://doi.org/10.1016/s10105182(98)80007-3 (PMID: 9702635)

3. Heidemann W, Terheyden H, Louis GK (2001) Analysis of the osseous/metal interface of drill-free screws and self-tapping screws. J Maxillofac Surg 29(2):69-74

4. Goelzer JG, Avelar RL, De Oliveira RB, Hubler R, Silveira RL, Machado RA (2010) Self-drilling and self-tapping screws: an ultrastructural study. J Craniofac Surg 21(2):513-515

5. Sowden D, Schmitz JP (2002) AO self-drilling and self-tapping screws in rat calvarial bone: an ultrastructural study of the implant interface. J Oral Maxillofac Surg 60(3):294-299

6. Bolm I, Goetze E, Kämmerer PW, Sader R, Klos M, Landes C et al (2017) Self-drilling and self-tapping miniscrews for osteosynthesis fixture after LeFort I osteotomy: an ex vivo trial for primary stability and a randomized clinical study. J Surg Res 212:246-252

7. Porto MA, Silva P, Rosa R, Volpon JB, Shimano AC, De Paula FJA et al (2012) Experimental in vivo acute and chronic biomechanical and histomorphometrical comparison of self-drilling and self-tapping anterior cervical screws. Eur Spine $\mathbf{J}$ 21:956-963

8. Wu X, Deng F, Wang Z, Zhao Z, Wang J (2008) Biomechanical and histomorphometric analyses of the osseointegration of microscrews with different surgical techniques in beagle dogs. Oral Sug Oral Med Oral Pathol Oral Radiol Endod 106(5):644-650

9. Heidemann W, Gerlach KL (1999) Clinical applications of drill free screws in maxillofacial surgery. J Craniomaxillofac Surg 27(4):252-255

10. Yadav S, Upadhyay M, Liu S, Roberts E, Neace WP, Nanda R (2012) Microdamage of the cortical bone during mini-implant insertion with self-drilling and self-tapping techniques: A randomized controlled trial. Am J Orthod Dentofac Orthop 141(5):538-546

11. Löhr J, Gellrich NC, Büscher P, Wahl D, Rahn BA (2000) Comparative in vitro studies of self-boring and self-tapping screws Histomorphological and physical-technical studies of bone layers. Mund Kiefer Gesichtschir 4(3):159-63

12. Alpert B, Gutwald R, Schmelzeisen R (2003) New innovations in craniomaxillofacial fixation: The 2.0 lock system. Keio J Med 52(2):120-7

13. Augustin G, Zigman T, Davila S, Udilljak T, Staroveski T, Brezak D et al (2012) Cortical bone drilling and thermal osteonecrosis. Clin Biomech (Bristol, Avon) 27(4):313-325

14. Allan W, Williams ED, Kerawala CJ (2005) Effects of repeated drill use on temperature of bone during preparation for osteosynthesis self-tapping screws. Br J Oral Maxillofac Surg 43(4):314-319

15. Widar F, Kashani H, Kanagaraja S, Dahlin C, Rasmusson L (2012) A retrospective evaluation of iatrogenic dental root damage with predrilled vs drill-free bone anchor screws for intermaxillary fixation. Dent Traumatol 28(2):127-131

16. Roccia F, Rossi P, Gallesio C, Boffano P (2009) Self-tapping and self-drilling screws for intermaxillary fixation in management of mandibular fractures. J Craniofac Surg 20(1):68-70

17. Schimming R, Gellrich N, Gutwald R, Schon R, Schmelzeisen R (1999) Self-drilling screws: clinical experiences in cranio and maxillofacial surgery. Int J Oral Maxillofac Surg 28(Suppl 1):26

Publisher's Note Springer Nature remains neutral with regard to jurisdictional claims in published maps and institutional affiliations. 